\title{
LES prediction of space-time correlations in turbulent shear flows
}

\author{
Li Guo · Dong Li · Xing Zhang · Guo-Wei He
}

Received: 2 May 2012 / Revised: 10 May 2012 / Accepted: 11 May 2012

(C)The Chinese Society of Theoretical and Applied Mechanics and Springer-Verlag Berlin Heidelberg 2012

\begin{abstract}
We compare the space-time correlations calculated from direct numerical simulation (DNS) and large-eddy simulation (LES) of turbulent channel flows. It is found from the comparisons that the LES with an eddy-viscosity subgrid scale (SGS) model over-predicts the space-time correlations than the DNS. The overpredictions are further quantified by the integral scales of directional correlations and convection velocities. A physical argument for the overprediction is provided that the eddy-viscosity SGS model alone does not includes the backscatter effects although it correctly represents the energy dissipations of SGS motions. This argument is confirmed by the recently developed elliptic model for space-time correlations in turbulent shear flows. It suggests that enstrophy is crucial to the LES prediction of spacetime correlations. The random forcing models and stochastic SGS models are proposed to overcome the overpredictions on space-time correlations.
\end{abstract}

Keywords Large-eddy simulation - Subgrid scale model · Space-time correlation · Turbulent shear flows

\section{Introduction}

Large-eddy simulation (LES) is designed to explicitly calculate the large-scale unsteady motions in turbulent flows whereas the effects of small-scale motions on large-scale ones are modeled using sub-grid scale (SGS) models. Therefore, it is expected that LES could accurately and reliably

The project was supported by the National Basic Research Program of China (973 Program) (2007CB814800) and the National Natural Science Foundation of China (10325211 and 10628206).

L. Guo $\cdot$ D. Li $\cdot$ X. Zhang · G.-W. He (莐

LNM, Institute of Mechanics,

Chinese Academy of Sciences,

100190 Beijing, China

e-mail: hgw@lnm.imech.ac.cn predict the unsteady motions at large scales [1]. The development of LES has showed the great potential of LES to predict the non-equilibrium properties of unsteady motions, with successful evidences [2]. These non-equilibrium properties are induced by either turbulence flows or their interaction with other physical processes such as turbulencechemistry interaction. Two typical examples are turbulencegenerated noise [3] and turbulent mixing [4]. In the first example, the acoustic intensity radiated by a turbulent flow is dependent on the two-time, two-point correlations of velocity fluctuations in the Eulerian frame [5-7]; in the second example, turbulent transport processes are naturally described by particle dispersion [8], or at least two-time velocity correlations of two particles in the Lagrangian frame [9]. The twotime, two-point correlations of velocity fluctuations in either Eulerian or Lagrangian frame are conventionally called as space-time correlations. They are the essential requirements for LES to correctly predict the non-equilibrium properties of unsteady motions in turbulent flows. Meanwhile, the impact of unsteady motions at large scales to small scale motions are also very important. The space-time correlations approach represents the preliminary work on those problems aligned in this direction.

A sub-grid scale (SGS) model is central to the LES prediction of space-time correlations. The simplest but mostlyused SGS model is the eddy-viscosity or Smagorinsky SGS model [10]. This model relates the residual stress to the filtered rate of strain and thus, it plays the role of energy sink to dissipate the extra energy at large scales. Therefore, the Smagorinsky model can correctly predict the energy spectra but it may not be able to correctly predict space-time correlations, since a space-time correlation can not be fully determined by energy spectra alone. In fact, small-scale motion makes two contributions to large-scale motion in turbulent flows [11]: energy dissipation and random backscatter. The random backscatter de-correlates large-scale motion and thus reduces the large-scale correlation length scales in both space and time. The standard Smagorinsky model represents the energy dissipation but ignores the random backscatter. 
Therefore, it may not be able to correctly predict the spacetime correlations. Space-time correlation is the simplest twotime, two-point statistical quantity. It represents the function of random backscatter from small-scale motions to largescale ones. The space-time correlation requires a higher level closure beyond one-time, two-point (spectral) closure and one-time, one-point closure in turbulence modeling theory.

The present study focuses on the Eulerian space-time correlations. Previous work on isotropic turbulence has found that LES with an eddy-viscosity SGS model could over-predict decorrelation time scales of space-time correlations and under-predict their magnitudes $[6,7]$. These results are supported by the sweeping hypothesis $[7,12]$ : the spacetime correlations in isotropic turbulence are mainly determined by energy spectra. Therefore, the under-predictions of LES on energy spectra result in larger decorrelation time scales and smaller correlation magnitudes. Park et al. [13] find the consistent results that the deterministic SGS models lead to slower decorrelations of space-time correlations in isotropic turbulence. They further propose this quantity to serve as the second requirement for the best deterministic SGS model, which is a powerful diagnostics character for SGS models. Dong and Sagaut [14] use the lattice Boltzmann method (LBM) to investigate the effects of SGS modeling on space-time correlations. Their result shows that there exist the distinct discrepancies in space-time correlations between the LES with the LBM-SGS models and the DNS. This implies that space-time correlations remain a challenge for this numerical method. The similar results are also observed in compressible boundary layers [15]. Those researches show that the LES with the standard Smagorinsky SGS model is not able to accurately predict the space-time correlations.

The present paper is devoted to study the LES prediction of space-time correlations in turbulent shear flows. The results on space-time correlations in isotropic turbulence can not be directly used to turbulent shear flows, since the mean shear rate in a turbulent shear flow provides another time scale in addition to the eddy turn-over time scale in isotropic turbulence. Favier et al. [16,17] investigate the space-time correlations in rotating turbulence. The rotation provides one more time scale different from isotropic turbulence and plays the role different from the shear rate. In the present study, we will take turbulent channel flow as our working case, since it is the typical example of turbulent shear flows with intensive study on their spatial statistics. We will compare the space-time correlations obtained from DNS and LES of turbulent channel flows. To quantify their disparity, we introduce a new measurement, directional correlation, and calculate its integral scale. The disparity is analyzed by the recently developed elliptic model $[18,19]$. The elliptic model implies that the space-time correlations in turbulent shear flows are determined by the energy spectra and two characteristic speeds: propagation velocity and sweeping velocity. A comparison is made between the propagation velocities from LES and DNS. Finally, we discuss the recently developed SGS models especially for space-time correlations.

\section{DNS and LES of turbulent channel flows}

Both DNS and LES of turbulent channel flow are performed on a staggered mesh using the finite volume method. The scheme is second order in space, using central differencing for both the convective and the viscous terms. For time advancing, a forth-order Runge-Kutta scheme is used for the convective term; while a third order implicit Runge-Kutta scheme is used for the viscous term. The Reynolds number based on the friction velocity and half channel width is 180 . The dimensions of channel are $4 \pi h, 2 h$ and $4 \pi h / 3$ in the streamwise, wall-normal and spanwise direction, respectively. A periodic boundary condition is applied in the streamwise and spanwise directions. The grid number is $130 \times 130 \times 130$ in DNS and $66 \times 66 \times 66$ in LES. We will find that the LES results are distinctly different from the DNS ones although the grid number in LES is about a half of the one in DNS. The grids are uniform in the streamwise and spanwise direction and are clustered in the normal direction near the solid walls. The governing equations are solved down to the wall using the Smagorinsky SGS model. The wall-nearest grid points are located at $y^{+}=0.29$ and $y^{+}=0.6$ for DNS and LES, respectively. A dynamic Smagorinsky model is used in the present simulation where the Smagorinsky coefficient is kept non-negative.

Figure 1 shows the energy spectra of streamwise velo-
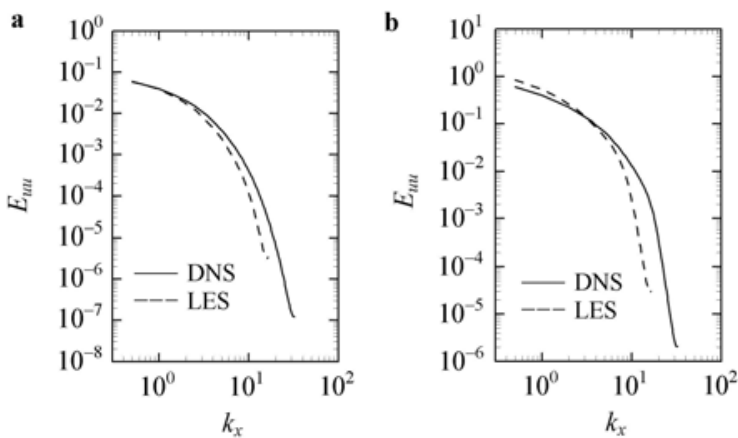

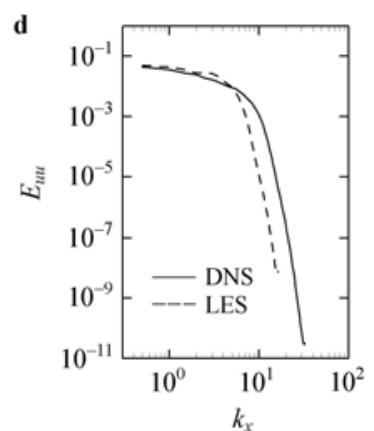

Fig. 1 The energy spectra of streamwise velocities. a $y^{+}=2.08 ; \mathbf{b} y^{+}=19.75 ; \mathbf{c} y^{+}=96.08 ; \mathbf{d} y^{+}=180$ 
city fluctuations at various wall regions and layers: $y^{+}=2.08$ (viscous sublayer), $y^{+}=19.75$ (buffer layer), $y^{+}=96.08$ (log-law region) and $y^{+}=180$ (outer region). The energy spectra from DNS decay over several decades without energy pile-up at higher wavenumbers. The energy spectra in LES approximately follow the trends of those in DNS but decay faster than DNS especially at higher wavenumbers. That is because the Smagorinsky SGS model used in the present study dissipates more energy at higher wavenumbers than DNS.

\section{Space-time correlation in turbulent channel flows}

We will consider the space-time correlations of velocity fluctuations in the streamwise direction

$R(r, \tau ; y)=\frac{\left\langle u_{1}(x+r, y, z, t+\tau) u_{1}(x, y, z, t)\right\rangle}{\left\langle u_{1}^{2}(x, y, z, t)\right\rangle}$,

where $r$ and $\tau$ are the space and time separations, respectively. The ensemble averaging is taken as the averaging in the streamwise and spanwise directions ( $x$ and $z$, respectively) and time $t$, since the streamwise component is spatially homogeneous in the directions of $x$ and $z$ and stationary in time. The DNS and LES data in Sect. 2 will be used to evaluate the space-time correlations.
A space-time correlation $R(r, \tau ; y)$ at a given location $y$ is the two-dimensional surface dependent on spatial separation $r$ and temporal delay $\tau$. To facilitate the comparison of the two-dimensional surfaces, we consider the space-time correlations taken in the direction of straight line $r=\tau \cdot \tan \alpha$ $(0 \leq \alpha \leq 2 \pi)$ at the locations $y^{+}=2.08$ (viscous sublayer), $y^{+}=19.75$ (buffer layer), $y^{+}=96.08$ (log-law region) and $y^{+}=180$ (outer region). Figures 2-4 plot the directional correlations, respectively, along the straight lines $\tau=0$ (space correlation), $r=0$ (time correlation) and $r=U \tau$ (convection direction), where $U$ is a convection velocity. Both LES and DNS results are shown on the same figures for our comparison. The spatial separation is normalized by the viscous length and the time delay normalized by the viscous time scale. It is observed from those three sets of figures that the correlation functions in DNS decorrelate slower in the viscous wall region than those in the outer layer. The LES approximately predicts the decorrelation properties of DNS but the correlations in LES decay slower than those in DNS. This is consistent with the previous observations in isotropic turbulence $[6,7,13]$ and turbulent channel flows [20,21]. Moreover, the discrepancies between DNS and LES are larger in the convection direction than those in other two directions, especially in the viscous wall region.
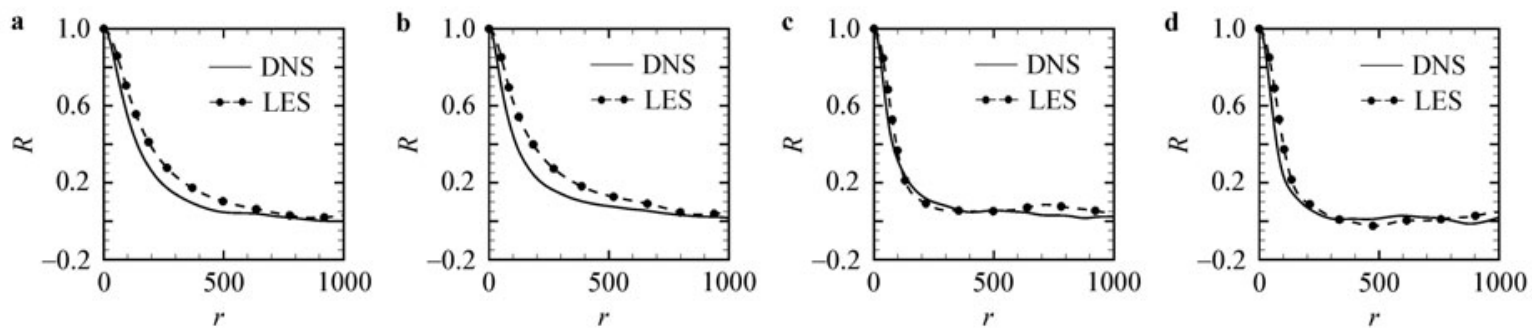

Fig. 2 The space correlations of streamwise velocities versus spatial separations. a $y^{+}=2.08$; $\mathbf{b} y^{+}=19.75 ; \mathbf{c} y^{+}=96.08 ; \mathbf{d} y^{+}=180$
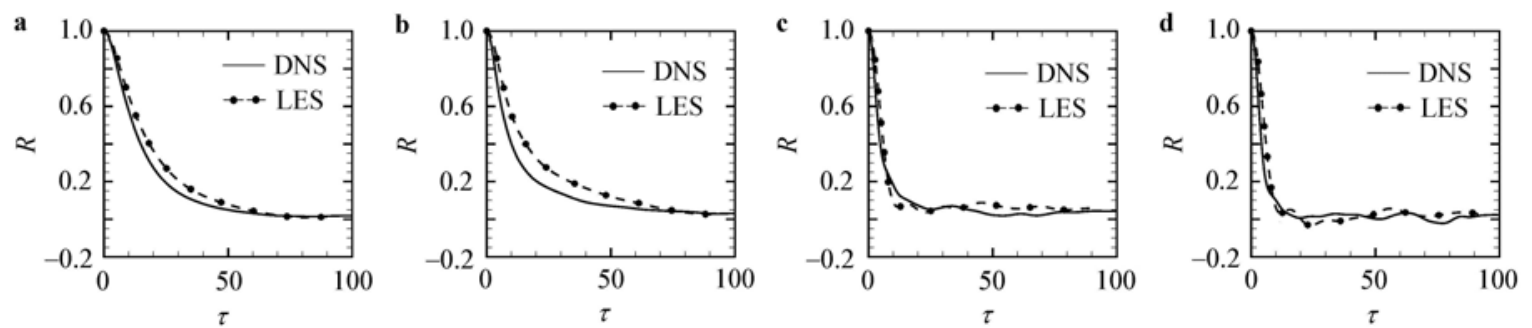

Fig. 3 The time correlations of streamwise velocities versus time separations. a $y^{+}=2.08 ; \mathbf{b} y^{+}=19.75 ; \mathbf{c} y^{+}=96.08 ; \mathbf{d} y^{+}=180$
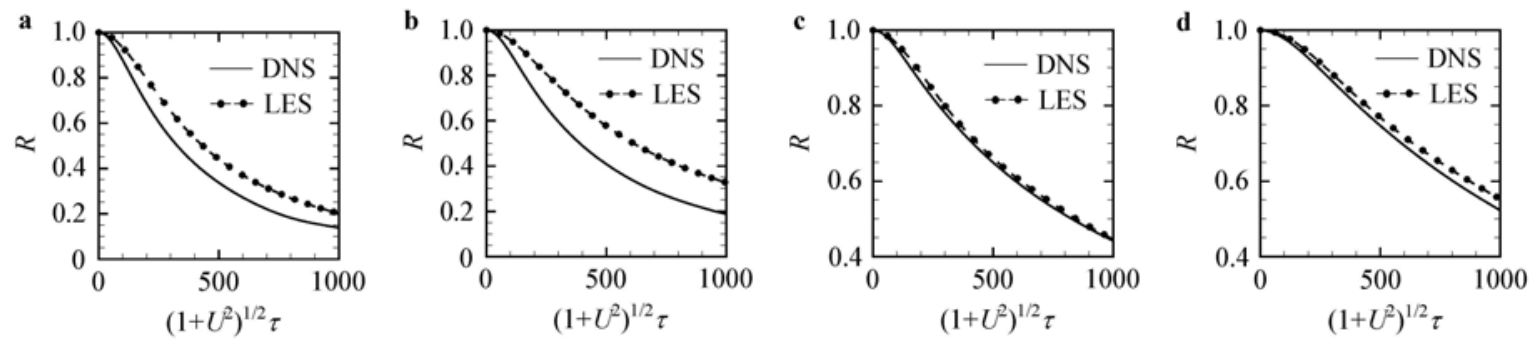

Fig. 4 The directional correlations of streamwise velocities in the propagation direction. The separation distance is $\sqrt{r^{* 2}+\tau^{* 2}}$, where the convection velocity is defined as $U=r^{*} / \tau^{*}$. a $y^{+}=2.08 ; \mathbf{b} y^{+}=19.75 ; \mathbf{c} y^{+}=96.08 ; \mathbf{d} y^{+}=180$ 
Figure 5 plots the iso-contours of space-time correlations for $y^{+}=2.08$ (viscous sublayer), $y^{+}=19.75$ (buffer layer), $y^{+}=96.08$ (log-law region) and $y^{+}=180$ (outer region). These curves look like elliptic with their preference directions. Their shapes are elongated with small aspect ratios. To visualize the distinctions between DNS and LES, we only plot the iso-correlations with the levels 0.5 and 0.9 . Evidently, the contours for LES are always outside of the ones for DNS. It indicates that the correlations from LES decay slower than those from DNS. Therefore, LES over-predicts the space-time correlations compared with DNS.

We plot in Fig. 6 the integral scales of directional correlations, $R(r, \tau)$ with $r=\tau \cdot \tan \alpha$ and $0 \leq \alpha \leq 2 \pi$, in the different regions. The directional correlation is defined as the space-time correlation evaluated in a straight line $r=\tau \cdot \tan \alpha$. The integral scales of directional correlations can be calcu-
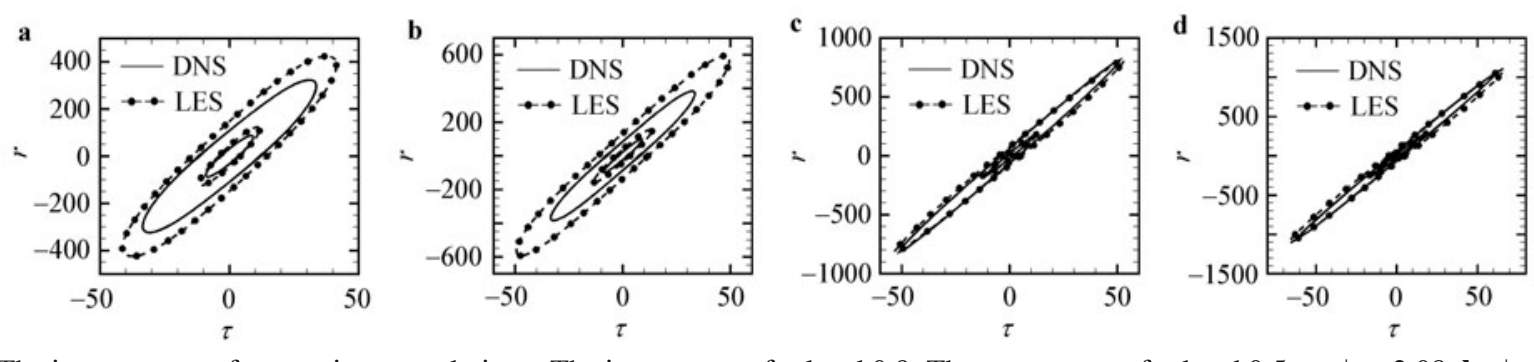

Fig. 5 The iso-contours of space-time correlations. The inner curves for level 0.9; The outer curves for level 0.5. a $y^{+}=2.08 ; \mathbf{b} y^{+}=19.75$; c $y^{+}=96.08 ; \mathbf{d} y^{+}=180$
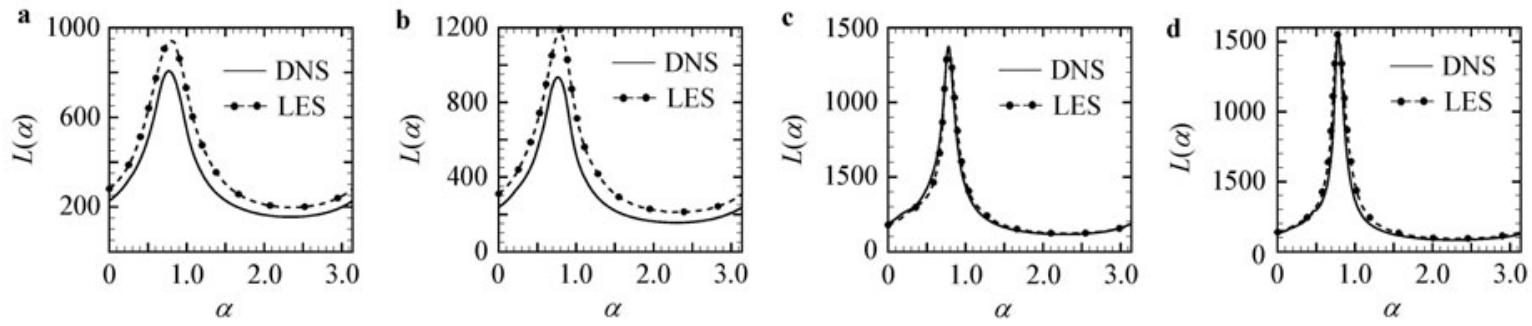

Fig. 6 The integral scales of the directional correlations at a $y^{+}=2.08 ; \mathbf{b} y^{+}=19.75 ; \mathbf{c} y^{+}=96.08 ; \mathbf{d} y^{+}=180$

Figure 7 compares the convection velocities obtained from the DNS and LES. The convection velocities are calculated in terms of the conventional definition [21,22]

$U=-\left.\frac{\partial^{2} R(r, \tau) / \partial r \partial \tau}{\partial^{2} R(r, \tau) / \partial r^{2}}\right|_{r=0, \tau=0}$.

We find that the convection velocities in LES are qualitatively consistent with the ones in DNS. However, the convection velocities in LES are larger than those in DNS in the near-wall region but smaller in the outer region. Recently, the convection velocity has been used to study the propagation of very long structures in turbulent channel flows [23]. The very long structures are associated with the $k_{x}^{-1}$ scaling of one-dimensional longitudinal spectrum at lower wavenumbers [24]. This result implies that LES can consistently predict the propagation velocity of coherent structures with small discrepancies.

lated from

$L(\alpha)=\int_{r=\tau \cdot \tan \alpha} R(r, \tau) \mathrm{d} \tau$.

It quantifies the decorrelation length scale of a space-time correlation taken in a given direction and describes how long a turbulent structure takes to decorrelate in the moving frame with velocity $\tan \alpha$. The directional correlation in convection direction $r=U \tau$ is the so-called integral life gral scales has one maximum at its peak and one minimum at the bottom plateau. Evidently, the integral scales in LES are larger than those in DNS in the viscous wall region but are very close in the outer layer. The largest differences ntegral scales between LES and DNS are located at the maximums, which are corresponding to the convection directions. 


\section{Theoretical analysis and discussions}

The overprediction of LES on space-time correlations can be understood by the following physical arguments: the effects of unresolved scales on resolved scales can be represented by energy transfer and random backscatter. The eddy-viscositytype SGS models are designed to model the energy transfer from resolved scales to unresolved scales but fail to account for the random backscatter from unresolved scales to resolved scales. The absence of random backscatter in LES leads to that the LES fields are more correlated than the DNS fields. This implies that space-time correlations in LES exhibit larger decorrelation length scales.

The overprediction of LES on space-time correlations in turbulent channel flows can be further analyzed using the recently developed elliptic model $[18,19]$. The elliptic model implies that the space-time correlations in turbulent shear flows are mainly determined by the space correlations and two characteristic speeds: propagation velocity $U$ and sweeping velocity $V$

$R(r, \tau)=R\left(\sqrt{(r-U \tau)^{2}+V^{2} \tau^{2}}, 0\right)$.

In the case of homogeneous sheared flows, the propagation velocity and sweeping velocity can be expressed as follows

$U=U_{1}$,

$V^{2}=(S \lambda)^{2}+\left\langle v_{i}^{2}\right\rangle$,

where $U_{1}$ is the mean velocity, $S$ is the mean shear rate, $\lambda$ is the Taylor micro-scale in space and $\left\langle v_{i}^{2}\right\rangle$ is the total energy of fluctuation velocities. In comparison with the classic Taylor frozen flow model, the elliptic model takes into account the deformation of eddies by introduction of the sweeping velocity. Therefore, this model is also called as the nonfrozen flow model. In fact, this model is a second approximation to the iso-correlation contours while Taylor's model is a first approximation to the contours. The Taylor frozen flow model and Kraichnan sweeping model are the two extreme cases of the elliptic model at vanishing propagation velocity or sweeping velocity. The recent experiments on Rayleigh-Bènard convection investigate the performance of the elliptic model for space-time correlations [25, 26].

The elliptic model proposes that the space-time correlations in turbulent shear flows are determined by its space correlations and propagation and sweeping velocities. An LES with the eddy-viscosity SGS model can well-predict the energy spectra $E(k)$ and thus, it can well-predict the space correlations $R(r, 0)$. In the LES, the propagation velocity can be correctly estimated (see Fig. 7) but the sweeping velocities are largely under-estimated (see more discussions below). As a result, the equivalent separations $\sqrt{(r-U \tau)^{2}+V^{2} \tau^{2}}$ in the LES are smaller than the ones in the DNS. According to the elliptic model (4), the space-time correlations in the LES are larger than the ones in DNS. In fact, the sweeping velocity $V$ are dependent on the mean shear rates $S$, total energy $\left\langle v_{i}^{2}\right\rangle$ of velocity fluctuations and Taylor's micro-scale $\lambda$. The first two parameters can be correctly estimated but the third one is underestimated in the LES. Noting the definition of Taylor's micro-scales $\lambda^{-2}=\int_{0}^{k_{\max }} q^{2} E(q) \mathrm{d} q / \int_{0}^{k_{\max }} E(q) \mathrm{d} q$ ( $k_{\max }$ is the largest wavenumber resolved in either DNS or LES), we compare the energy and enstrophy in DNS and LES

$$
\begin{aligned}
& \int_{0}^{k_{\mathrm{c}}} E^{\mathrm{LES}}(q) \mathrm{d} q \approx \int_{0}^{k_{\max }} E^{\mathrm{DNS}}(q) \mathrm{d} q, \\
& \int_{0}^{k_{\mathrm{c}}} q^{2} E^{\mathrm{LES}}(q) \mathrm{d} q<\int_{0}^{k_{\max }} q^{2} E^{\mathrm{DNS}}(q) \mathrm{d} q,
\end{aligned}
$$

where $k_{\mathrm{c}}$ is the cutoff wavenumber in LES. This implies that the LES approximately predicts the total energy of DNS but under-estimate the enstrophy of DNS. Therefore, the LES overpredicts the Taylor micro scales. This result is consistent with the previous physical arguments. Meanwhile, it implies that enstrophy is crucial to the LES prediction of space-time correlations.

There exist two approaches to model the random backscatter to overcome the overprediction of LES on spacetime correlations. One way is to introduce a random force to represent the backscatter effect. He et al. [6] has developed a constrain for the random force in LES to recover the decorrelation length scales. Recently, Marstorp et al. [27] propose a stochastic Smagorinsky SGS model which allows for backscatter of the energy at unresolved scales. This model can reduce the overprediction of LES on space-time correlations especially in decaying isotropic turbulence.

\section{Conclusions and future work}

We find that the LES with the eddy-viscosity SGS models over-predicts space-time correlations than DNS in turbulent channel flows. This is qualitatively consistent with previous observations in isotropic turbulence $[6,7]$. Furthermore, we find that the LES overpredictions in turbulent channel flows, especially in the near-wall regions, are relatively larger than those in isotropic turbulence. The reasons for the LES overpredictions in isotropic turbulence can be provided with the random sweeping model $[12,28]$ : the space-time correlations in isotropic turbulence are mainly determined by its energy spectra. As a result, the LES under-prediction of energy spectra leads to its overprediction of space-time correlations. However, a space-time correlation in turbulent shear flows depends on both energy spectra and enstrophy. The in-accurate predictions of either energy spectra or enstrophy induce the larger over-estimations of space-time correlations.

We propose the directional correlations to describe the variation of space-time correlations along a straight line of slop $U$. The integral scales of directional correlations characterize the decorrelation length scales of turbulent structures in the moving frame with a speed $U$. The moving speed at which a directional correlation decay most slowly is the propagation velocity of coherent structures. The nu- 
merical comparisons in the present study show that LES approximately predicts the propagation velocity of the coherent structures but largely over-predict their integral scales. The latter implies that turbulent structures in the LES with the eddy-viscosity SGS models are larger in size than those in DNS.

The absence of backscatter in the eddy-viscosity SGS models is the main reason for LES overprediction of spacetime correlations. The eddy-viscosity SGS model could not represent the backscatter effect and generates a more correlated field. Therefore, the LES field exhibits larger length scales in both space and time. The deterministic SGS models are not able to include the backscatter effects and therefore, they are not able to correctly predict space-time correlations. Instead, the stochastic SGS models take backscatter into account and thus, they are able to recover the space-time correlations in DNS, such as random forcing mode [6,29], stochastic Smagorinsky model [27] and stochastic backscatter models [30,31].

\section{References}

1 Pope, S.B.: Turbulent Flows. Cambridge University Press, Cambridge, UK (2000)

2 Moin, P.: Advances in large eddy simulation methodology for complex flows. Int. J. Heat Fluid Flow 23, 710-720 (2002)

3 Wang, M., Freund, J.B., Lele, S.K.: Computational prediction of flow-generated sound. Annu. Rev. Fluid Mech. 38, 483-512 (2006)

4 Pitsch, H.: Large-eddy simulation of turbulent combustion. Annu. Rev. Fluid Mech. 38, 453-482 (2006)

5 Goldstein, M.E.: Aeroacoustics. McGraw-Hill, New York (1976)

6 He, G.W., Rubinstein, R., Wang, L.P.: Effects of subgrid-scale modeling on time correlations in large eddy simulation. Phys. Fluids 14, 2186-2193 (2002)

7 He, G.W., Wang, M., Lele, S.K.: On the computation of spacetime correlations by large-eddy simulation. Phys. Fluids 16, 3859-3867 (2004)

8 Flohr, P., Vassilicos, J.C.: A scalar subgrid model with flow structures for large-eddy simulation of scalar variance. J. Fluid Mech. 407, 315-349 (2000)

9 Yang, Y., He, G.W., Wang, L.P.: Effects of subgrid-scale modeling on Lagrangian statistics in large-eddy simulation. J. Turbulence 9, 1-25 (2008)

10 Smagorinsky, J.: General circulation experiments with the primitive equations. Mon. Wea. Rev. 91, 99-164 (1963)

11 Meneveau, C., Katz, J.: Scale-invariance and turbulence models for large-eddy simulation. Annu. Rev. Fluid Mech. 32, 1-32 (2000)

12 Kraichnan, R.H.: Kolmogorov's hypotheses and Eulerian turbulence theory. Phys. Fluids 7, 1723 (1964)
13 Park, N., Lee, S., Lee, J., et al.: A dynamic subgrid-scale eddy viscosity model with a global model coefficient. Phys. Fluid 18, 125109 (2006)

14 Dong, Y.H., Sagaut, P.: A study of time correlations in lattice Boltzmann-based large-eddy simulation of iso-tropic turbulence. Phys. Fluid 20, 035105 (2008)

15 Martin, P.: Preliminary study of the SGS time scales for compressible boundary layer using DNS data. AIAA paper 20050665

16 Favier, B., Godeferd, F.S., Cambon, C.: Modeling the far-field acoustic emission of rotating turbulence. J. Turbulence 9, 1-21 (2008)

17 Favier, B., Godeferd, F.S., Cambon, C.: On space-time correlations of isotropic and rotating turbulence. Phys. Fluids 22 015101-1 (2010)

18 He, G.W., Zhang, J.B.: Elliptic model for space-time correlations in turbulent shear flows. Phys. Rev. E 73, 055303 (2006)

19 Zhao, X., He, G.W.: Space-time correlations of fluctuating velocities in turbulent shear flows. Phys. Rev. E 79, 046316 (2009)

20 Quadrio, M., Luchini, P.: Integral space-time scales in turbulent wall flows. Phys. Fluid 15, 2219 (2003)

21 Kim, J., Hussain, F.: Propagation velocity of perturbations in turbulent channel flow. Phys. Fluid A 5, 695-706 (1993)

22 Choi, H., Moin, P.: On the space-time characteristics of wallpressure fluctuations. Phys. Fluids A 2, 1450-1460 (1990)

23 Chung, D., Mckeon, B.J.: Large-eddy simulation of large-scale structures in long channel flow. J. Fluid Mech. 661, 341-364 (2010)

24 Hutchins, N., Marusic, I.: Large-scale influences in near-wall turbulence. Phil. Trans. R. Soc. A 365, 647-664 (2007)

25 He, X.Z., He, G.W., Tong, P.: Small-scale turbulent fluctuations beyond Taylor's frozen flow hypothesis. Phys. Rev. E 81, 065303(R) (2010)

26 Zhou, Q., Li, C.M., Lu, Z.M., et al.: Experimental investigation of longitudinal space-time correlations of the velocity field in turbulent Rayleigh-Bènard convection. J. Fluid Mech. $\mathbf{6 8 3}$, 94-111 (2011)

27 Marstorp, L., Brethouwer, G., Johanssonc, A.V.: A stochastic subgrid model with application to turbulent flow and scalar mixing. Phys. Fluids 19, 035107 (2007)

28 Tennekes, H.: Eulerian and Lagrangian time microscales in isotropic turbulence. J. Fluid Mech. 67, 561 (1975)

29 Carati, D., Ghosal, S., Moin, P.: On the representation of backscatter in dynamic localization models. Phys. Fluid. 7, 606 (11 pages) (1995)

30 Mason, P., Thomson, D.J.: Stochastic backscatter in large-eddy simulation of boundary layers. J. Fluid Mech. 242, 51-78 (1992)

31 Westbury, P.S., Dunn, D.C., Morrison, J.F.: Analysis of a stochastic backscatter model for the large-eddy simulation of wall-bounded flows. Europ. J. Mech. B/Fluid 23, 735-758 (2004) 